\title{
JOVIAN DAM LINEAR POLARIZATION STUDY FROM COORDINATED, DISTANT, GROUND-BASED RADIO TELESCOPES
}

\author{
A. Lecacheux*, M. Imai ${ }^{\dagger}$, T. Clarke $\ddagger$ C. Higgins ${ }^{\S}$, M. Panchenko $\mathbb{I}$ \\ A. Konovalenkoll, and A. Brazhenko**
}

\begin{abstract}
Ground-based, complete measurement of Jovian DAM polarization ellipse is a difficult task, due to various terrestrial ionosphere effects encountered along the propagation path of the radiation: scintillations strongly modulate the received intensity, and the uncertainty on the (Faraday) rotation measure is of the same order of magnitude as the position angle to be measured.

Simultaneous observations of Jupiter from distant ground based radio telescopes, as those which were obtained in 2015-2016 by using LWA1 (USA), URAN2 (Ukraine) and Nançay Decameter Array (France), may solve this ambiguity, since the local ionosphere effects can, in principle, be disentangled from common Jovian radiation properties. The measurement method is discussed, in particular how each wideband polarimeter can be accurately calibrated and how the (Faraday) rotation measure can be confidently estimated.

Some preliminary results as well as their theoretical implications are discussed.
\end{abstract}

\footnotetext{
* CNRS - Observatoire de Paris, Meudon, France

$\dagger$ Department of Physics and Astronomy, University of Iowa, Iowa City, IA, USA

$\ddagger$ Remote Sensing Division, US Naval Research Laboratory, DC, USA

$\S$ Middle Tennessee State University, Murfreesboro, TN, USA

I Space Research Institute, Austrian Academy of Sciences, Graz, Austria

" Institute of Radio Astronomy, National Academy of Sciences of the Ukraine, Kharkov, Ukraine

**Institute of Geophysics, Gravimetric Observatory, Poltava, Ukraine
} 
